Universidad de Lima

Facultad de Psicología

Carrera de Psicología

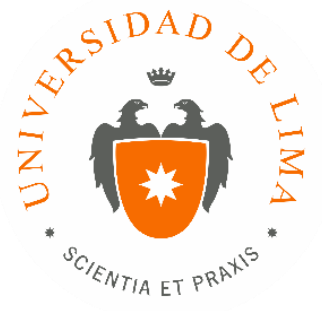

\title{
EXPERIENCIA DE TRABAJO EN TERAPIA PSICOLÓGICA INDIVIDUAL CON NIÑOS EN UN CENTRO PRIVADO DE LIMA METROPOLITANA
}

Trabajo de suficiencia profesional para optar el título profesional de Licenciado en Psicología

\section{Luciana del Carmen Lucar Flores}

Código 20100642

$$
\text { Lima - Perú }
$$

Febrero de 2019 



\section{EXPERIENCIA DE TRABAJO EN TERAPIA PSICOLÓGICA INDIVIDUAL CON NIÑOS EN UN CENTRO PRIVADO DE LIMA METROPOLITANA}




\section{TABLA DE CONTENIDO}

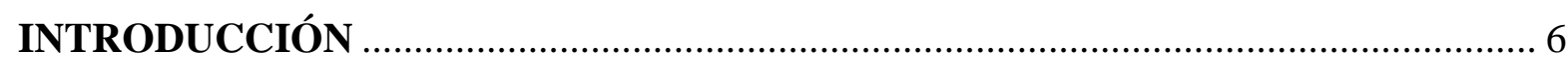

CAPÍTULO I: IDENTIFICACIÓN DEL PROBLEMA ............................................. 7

CAPÍTULO II: DESCRIPCIÓN DE LAS ACTIVIDADES Y TAREAS REALIZADAS

$2.1 \quad$ Identificación del motivo de consulta.......................................11

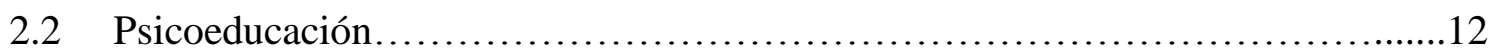

2.3 Estrategias para el cambio........................................... 13

CAPÍTULO III: RESULTADOS DE LA INTERVENCIÓN...................................16

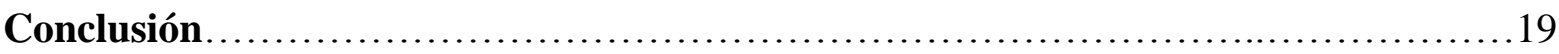

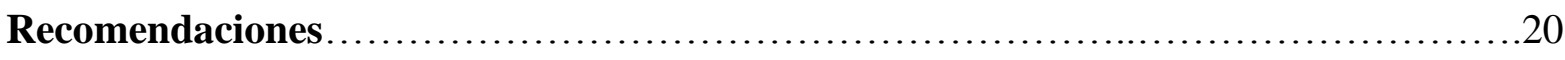

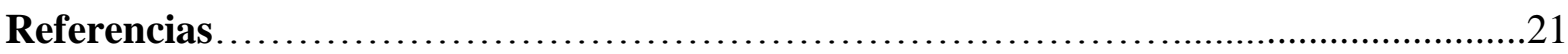




\section{INTRODUCCIÓN}

El presente reporte tiene por objetivo sustentar el dominio práctico y teórico del bachiller, aplicado en la experiencia laboral en el ámbito psicológico, específicamente en el área clínica. Dicha experiencia consiste en la intervención terapéutica individual con niños, realizada en un centro privado en el distrito de San Borja en Lima Metropolitana.

A continuación se expone brevemente el origen del problema, para lo cual se presentarán los conceptos de salud y salud mental, acompañados de la descripción de algunas características de la situación actual local, donde se puede identificar la demanda de atención psicológica existente y el poco acceso que las personas suelen tener a esta. Luego, se describirán las características principales de la institución donde se tuvo la experiencia laboral, así como el modelo teórico optado para abordar la intervención y los problemas más comunes que suelen presentarse.

Seguidamente, se procede a explicar el proceso realizado durante la intervención terapéutica, con el objetivo de contribuir a la resolución de algunos de los problemas presentados por la población atendida. Dichas actividades se dividen en tres partes, de acuerdo al procedimiento usualmente aplicado y siguiendo la metodología de trabajo de la institución.

Posteriormente, se realiza el análisis de los resultados obtenidos a partir de la intervención realizada. Especificando los cambios observados a modo general en los pacientes y algunas curiosidades identificadas durante la aplicación. Así como oportunidades de prevenir la problemática intervenida y temas relevantes para investigaciones posteriores.

Finalmente, se presentan las conclusiones más importantes sobre el trabajo realizado y el análisis del mismo. Seguido de algunas recomendaciones aplicables a la población intervenida como a la comunidad local en general. 


\section{CAPÍTULO I: IDENTIFICACIÓN DEL PROBLEMA}

"La salud es un estado de completo bienestar físico, mental y social, y no solamente la ausencia de afecciones o enfermedades" (Organización Mundial de la Salud [OMS], 2013, p.7). No es novedad que, muchas veces, las personas confundan el concepto salud y crean que el cuidado de la misma solo requiere asistir donde un especialista cuando se presente una molestia que impida continuar con la rutina diaria o que ponga en peligro la vida. Dejando de lado el verdadero mantenimiento de la salud como estado integral de bienestar.

La salud mental es concebida por la OMS (2013) como un estado de bienestar en el cual el individuo desarrolla sus capacidades, supera el estrés normal de la vida, trabaja de forma productiva y fructífera, aportando a su comunidad. En los niños, se resaltan los aspectos del desarrollo, el sentido positivo de la identidad, la capacidad para gestionar pensamientos y emociones, así como para crear relaciones sociales, la aptitud para aprender y adquirir una educación que los capacitará para participar activamente en la sociedad.

Siendo así que la salud mental puede verse afectada por una serie de factores que interactúan entre sí, como los socioeconómicos, políticos, legales o ambientales. Por ejemplo, en el Perú, la situación económica de un adulto suele ser consecuencia de la calidad de educación a la que pudo acceder combinada con la ubicación geográfica, los servicios accedidos y las amistades que logró establecer. Asimismo, la familia, educación y contexto en el que crecen será la fuente de aprendizaje del estilo de crianza que impartirán en sus hijos.

De la misma manera, según la información brindada por la OMS (2013), muchos trastornos mentales presentan tasas elevadas de discapacidad y mortalidad, presentando problemas de salud física que no suelen ser atendidos a tiempo a causa de la sintomatología de carácter psicológico. Según esta información, a nivel mundial, el suicidio es la segunda causa más frecuente de muerte en los jóvenes. La OMS indica que en los países de ingresos bajos y medios, como el Perú, gran porcentaje de las personas con trastornos mentales graves no 
reciben tratamiento (entre $76 \%$ y $85 \%$ ). Además, el número de profesionales especializados y generales que se ocupan de la salud mental es insuficiente.

Del mismo modo, la proporción de países que disponen de políticas, planes y legislación sobre la salud mental es mucho mayor entre los de ingresos elevados que entre los de ingresos bajos. Siendo asi que el número de personas amparadas por una legislación en materia de salud mental en paises de ingresos bajos equivale a un tercio en comparación con dicho número en países de ingresos elevados (OMS, 2013).

Esta situación se observa claramente en el Perú, donde, como menciona Alarcón (2015), cada vez se encuentra mayor prevalencia e incidencia de enfermedades mentales, lo cual se une a la escasez de profesionales de la salud mental con la preparación y competencia adecuada. A lo que se suma la baja proporción de presupuestos locales considerados para la atención, cuidado y prevención de este tipo de problemas, así como la insuficiente cobertura de seguros y existencia de espacios equipados para el servicio. Piazza y Fiestas (2015) al comentar sobre el estudio mundial de Salud Mental (EMSM) que se realizó en el año 2005, afirman que en este se encontró que del total de personas identificadas con desórdenes mentales en zonas urbanas del Perú, solo una minoría recibió tratamiento (19\%).

Siendo así que esta situación impulsó a que en el mes de marzo del año 2015 se funde la institución donde el bachiller realiza su experiencia laboral, la cual nace con el objetivo de contribuir en la promoción de la salud mental en niños, adolescente y adultos, potenciando y desarrollando sus habilidades, contribuyendo con su crecimiento personal. Para lo cual se recluta un equipo con profesionales especializados en diversas disciplinas relacionadas a la salud mental, contando con el espacio físico adaptado para la adecuada intervención individual y grupal. 
El centro se ubica en la urbanización Chacarilla del Estanque dentro del distrito de San Borja, en Lima. Está dirigido a niños y adultos del segmento socioeconómico medio y alto, siendo la mayoría de pacientes residentes de los distritos de Surco, La Molina, San Borja y Miraflores. En dicha institución, desde su fundación hasta la actualidad, se realizan intervenciones terapéuticas psicológicas, neuropsicológicas, de aprendizaje, de lenguaje y habla, sensorialocupacional y psicomotriz, tanto individuales como grupales. Así como charlas, talleres y asesorías en estilos de crianza y diversos temas de interés para la comunidad.

En la actualidad, según el registro de la administración del centro, se atiende un promedio de 75 pacientes al mes, de los cuales aproximadamente 60 son niños (R. Orlandini, comunicación personal, 6 de febrero de 2019). Quienes, en su mayoría, llegan por derivación de profesionales de la salud, docentes o por algún conocido que recomendó el lugar. También hay un porcentaje menor de pacientes que se acercan debido a la publicidad en redes sociales. Asimismo, como parte del compromiso con el desarrollo de la comunidad, al menos dos veces al año se realizan despistajes en el área neuropsicológica, psicológica, de aprendizaje y de lenguaje y habla en lugares de escasos recursos. Además, se le invita a cada profesional trabajar en una intervención individual gratuitamente durante el año.

En la institución y a título personal, no solo se busca tratar patologías severas que cumplan con criterios diagnósticos sino trabajar en el mantenimiento y promoción de la salud mental. Interviniendo a tiempo y brindándole a la persona las herramientas necesarias para resolver y prevenir dificultades mayores, ayudando en que logre un desempeño funcional en todos los ámbitos en las diferentes etapas de su vida.

Cabe mencionar que, dentro de la psicología, existen modelos terapéuticos con diferentes concepciones sobre el ser humano y lo que es una patología. En la institución se tiene en claro las limitaciones de cada modelo, por lo que se considera esencial contar con 
profesionales de distintos enfoques a fin de realizar un trabajo complementario teniendo como prioridad el bienestar del paciente.

Para fines de este reporte se aplicará el modelo de Terapia Racional Emotivo Conductual (TREC) planteada por Albert Ellis en 1962. Browne, Dowd y Freeman (2010) explican que la TREC es un modelo cognitivo donde los problemas emocionales son el resultado de creencias irracionales. Las cuales se definen como pensamientos evaluativos carentes de fundamento lógico, empírico o dogmático. El modelo identifica algunos pensamientos irracionales nucleares, incluyendo en ellos a las demandas (creencia de que las cosas deben ser absolutamente de una forma u otra), la catastrofización (creencia de que cuando algo sale mal es lo más terrible que podría pasar), escasa tolerancia a la frustración (creer que cuando las cosas no salen según nuestro deseo no lo podremos soportar) y finalmente, la valoración global (juzgar a personas otorgándoles un valor global de acuerdo a su conducta). Asimismo, se encuentran los pensamientos racionales, que se caracterizan por ser preferencias o deseos, siendo estos pensamientos moderados los que no resultan en perturbaciones emocionales. Siendo así que la TREC define la patología en función al modelo ABC. En el cual la A representa el evento activador, la $\mathrm{B}$ es la creencia relacionada al evento y la $\mathrm{C}$ es la emoción o consecuencia conductual a partir de la creencia (Browne et al., 2010; Dryden, 2009). De manera que no es el evento el que causa la emoción o conducta del individuo, sino los pensamientos y creencias que se activan a partir de ello.

En la institución donde se realizó la experiencia de trabajo comúnmente suelen asistir niños con motivos de consulta asociados a dificultades conductuales que repercuten en un desempeño disfuncional en el área social, académica o familiar. Se identifica que esto se relaciona con problemas para tolerar la frustración y regular sus impulsos, decaimiento emocional ligado a dificultades para lograr la aceptación incondicional, escasa asertividad y altos niveles de ansiedad. 


\section{CAPÍTULO II: DESCRIPCIÓN DE LAS ACTIVIDADES Y}

\section{TAREAS REALIZADAS}

Para fines del presente reporte se expondrán aquellas actividades realizadas en intervención psicológica individual de los casos asignados por la dirección con niños entre 4 y 10 años. Sin considerar las intervenciones individuales del área de aprendizaje, así como talleres y distintas modalidades grupales de intervención realizadas por la bachiller en mención.

\subsection{Identificación del motivo de consulta}

En la institución, al aplicar una intervención psicológica individual con niños, se sigue un esquema de trabajo que consiste en programas de 25 sesiones, donde la primera es una entrevista inicial con los padres para conocer el motivo de consulta y los antecedentes más relevantes (material propio de la institución).

Luego se dan cuatro sesiones con el niño para conocerlo, observar las dificultades manifestadas por los padres e identificar recursos y otras áreas de conflicto. Se realizan actividades lúdicas con el objetivo de romper el hielo y para que logre compartir lo que le molesta o preocupa, generando así un vínculo de confianza entre el terapeuta y el niño. Además, se busca conocer el motivo de consulta desde su perspectiva y posibles fantasías sobre el proceso. Se usa la metáfora del doctor, explicando que "un psicólogo ayuda a curar problemas, miedos y molestias con diferentes herramientas que no son remedios". Se comparten historias de otros niños que asistieron a terapia para ayudarlo a normalizar su problema y generar esperanza de cambio.

Es usual que los niños presenten motivos de consulta relacionados a un desempeño disfuncional en algún área de su vida. Por lo cual, se remite un cuestionario al 
centro educativo (material propio de la institución) para ser completado por su tutor y/o psicólogo del grado, a fin de recibir información complementaria.

Al terminar esta primera fase se tiene otra reunión con los padres para explicar el plan de intervención aprobado por dirección (material propio de la institución). Es importante contar con el compromiso de los padres en el acompañamiento emocional y en la práctica de las pautas o estrategias de intervención.

\subsection{Psicoeducación}

Antes de empezar a trabajar el problema específico con el niño es importante que conozca cómo funciona la mente humana y las emociones, brindándole información acorde a su edad. Primero, se le facilita un vocabulario emocional y a través de láminas con caras que expresan emociones, cuentos, videos, entre otras actividades se ejercita el reconocimiento de estas en los demás para luego identificarlas en sí mismo. Se utiliza el termómetro emocional para explicar las diferentes intensidades emocionales y normalizar las emociones negativas.

Seguidamente, se le enseña en qué consiste el modelo de terapia a trabajar. Según DiGiuseppe, Doyle, Dryden y Backx (2014), el procedimiento base para la intervención TREC consta de 5 pasos principales conocidos como "ABCDE”. El primero (A) consiste en identificar el evento activador, preguntando por un problema específico e invitando al paciente a narrarlo a detalle. Es crucial que el niño trate de recordar una experiencia reciente para tener detalles certeros.

Luego, se procede a determinar y medir las consecuencias emocionales y conductuales del problema (C) notando qué emociones y conductas presenta la persona, con qué intensidad, duración y qué repercusiones trae consigo (DiGiuseppe et al., 2014). Al haber trabajado una psicoeducación emocional, les suele resultar fácil identificar tanto las emociones como sus conductas asociadas. 
Sin embargo, puede costar comprender las repercusiones que tienen en el ambiente. Por ejemplo, a un niño se le llama la atención en clase, se pone triste y llora, es probable que logre reconocer la emoción de tristeza y la conducta de llorar, pero le costará más trabajo identificar consecuencias en su entorno, como la distracción que genera en sus compañeros. Es por ello que a eso se le dedica más tiempo y se emplean materiales según la edad del niño, como hojas de registro o dibujos.

Después, se evalúan las creencias del paciente (B), a fin de identificar las irracionales que alimentan el problema (DiGiuseppe et al., 2014). Se busca acompañar el discurso verbal con juegos de roles o dibujos. Dependiendo de la edad se da mayor énfasis a lo verbal o no verbal. Por ejemplo, será más fácil que un niño de 5 años logre identificar los pensamientos que surgen en una situación al recrearla con muñecos o dibujándola, en lugar de usar solo el recurso verbal. Con niños mas grandes se utiliza de forma complementaria el registro de pensamientos como tarea para realizar durante la semana en casa.

Cuando ya se tienen en claro A, B y C, se explica cómo funciona la relación entre ellos (DiGiuseppe et al., 2014). Se emplean cuentos, láminas con situaciones y metáforas para aclarar que la situación en sí no es la que genera la emoción, sino las creencias que la acompañan, luego para verificar si lo comprende se solicita que lo explique en sus propias palabras para luego trasladarlo a algún ejemplo personal.

\subsection{Estrategias para el cambio}

Posteriormente, se inicia la discusión de las creencias y evaluaciones irracionales (D) (DiGiuseppe, et al., 2014). Con los niños, por su nivel de desarrollo cognitivo, se utiliza un nivel de discusión concreto y específico de estilo didáctico. Donde se analizan las desventajas de las creencias en cuestión y evidencias cotidianas que las refuten, invitándolos a ser "detectives de pensamientos". 
Después se formula con el niño un pensamiento racional alternativo (DiGiuseppe, et al., 2014). Por ejemplo, "sacarme C en mi examen es terrible y no soportaré volver al colegio", se transforma a: "sacarme C en mi examen no me gusta, preferiría una A, pero sé que no es terrible y podré ir al colegio". Las cuales se formulan como auto-instrucciones a practicar y recordar en situaciones futuras.

Finalmente, para lograr un cambio profundo (E), se busca aplicar estrategias específicas según cada caso (DiGiuseppe, et al., 2014). Las cuales se indican a continuación según la problemática más recurrente.

En cuanto a la aceptación incondicional, Browne, et al. (2010) indican que esta consiste en deslindar la valoración de las personas de sus resultados, conductas o rendimientos. Para lograrlo se elabora una lista con todas sus características personales (tanto positivas como negativas) para luego otorgarles igual de importancia y reconocer que juntas lo hacen ser único y especial. Del mismo modo se fomentan actividades que no realizaba por temor a fracasar y ser juzgado, así como se le encomienda la tarea de investigar sobre personajes que considere admirables e identificar dificultades o errores que pudieron cometer.

Asimismo, es usual la intolerancia a la frustración. Browne, et al. (2010) refieren que surge de las exigencias por aquello que es fácil, rápido y cómodo. Para realizar un cambio se proponen sesiones con secuencias de actividades predeterminadas buscando retar al niño y poner en práctica las nuevas creencias. Cuando se observa que se siente incómodo o se niega a realizar una actividad, se reconoce la emoción y el hecho de que él preferiría sentirse de otra manera. La misma reacción se recomienda a los padres en casa, así como no ceder ante las rabietas. Además, se aplican estrategias como el "termómetro de catástrofes" y se grafican o escriben las auto-instrucciones para que las recuerde y pueda modificar su conducta. 
Otra dificultad recurrente es la ansiedad, observada en la constante evitación o escape en situaciones valoradas irracionalmente como "amenazas", estando en estado de alerta constante y mostrándose susceptibles ante los cambios (Dryden, 2009). Para cambiar esta valoración primero se le enseñan algunas estrategias de auto regulación como la relajación muscular, control respiratorio y la imaginación para visualizar una situación que le permita calmarse. Luego se enlistan situaciones que le generan ansiedad, con las que se inicia la exposición progresiva aplicando la auto regulación y celebrando cada logro como motivación para continuar.

Por último, las creencias mencionadas suelen relacionarse con dificultades en la interacción social. Muchos niños llegan al centro por haberse visto involucrados en peleas con sus compañeros o haber sido rechazados, sin saber cómo actuar cuando alguien hace algo que les incomoda. Según lo recomendado por Ellis y Tafrate, (2013), para brindarle herramientas se realiza un entrenamiento práctico donde se explica, a través de metáforas, en qué consiste la comunicación pasiva, agresiva y asertiva. Analizando las ventajas de la comunicación asertiva y practicando cómo aplicarla a través del juego de roles.

Es importante mencionar que los problemas señalados no se presentan de forma aislada, ya que un pensamiento irracional genera consecuencias diversas y se manifiesta en varios contextos. Es por ello que para alcanzar los objetivos, se debe integrar las creencias que suele tener el niño en diferentes escenarios de su vida. Finalmente, según el esquema de trabajo de la institución, al llegar a la sesión 24 se realiza un informe de lo trabajado. Este se entrega a los padres en una sesión donde se determina la continuidad o no de la terapia. A veces solo se requiere un programa para lograr los objetivos, pero en ocasiones es necesario continuar con la terapia enfocados en estrategias específicas y pautas para el hogar. 


\section{CAPÍTULO III: RESULTADOS DE LA INTERVENCIÓN}

Durante el año 2018 el autor de este reporte atendió nueve niños en intervención terapéutica psicológica individual que asistieron con una frecuencia de dos veces por semana, completando un total de 675 sesiones acorde al esquema de trabajo de la institución y siguiendo el modelo de Terapia Racional Emotivo Conductual (TREC). Se siguió dicho modelo ya que el bachiller en mención ha completado el programa de formación en una escuela en Lima certificada por el Albert Ellis Institute de Nueva York, para aplicarlo de forma adecuada.

En la mayoría de los casos, los niños presentan dificultades para lograr la aceptación incondicional de sí mismos. A partir de la intervención se alcanzaron cambios positivos donde los pacientes lograron reconocer y valorar sus habilidades, sin embargo en muchas ocasiones continuaba dándole una valía exagerada a aquellos puntos negativos de su personalidad. Se observó que en los casos en que los padres se involucraban más en la terapia y participaban activamente, siguiendo las pautas recomendadas, validando las diversas emociones de sus hijos y comunicándose asertivamente con ellos; se lograba un cambio más profundo. Donde los niños realmente lograban una aceptación incondicional no solo de sí mismos sino de los demás también, lo cual se veía reflejado en su motivación hacia el aprendizaje y desarrollo de su creatividad, así como en el uso adecuado de las herramientas de asertividad aprendidas en el espacio terapéutico. Lo cual tiene como consecuencia la consolidación de vínculos más saludables con sus pares.

Con respecto a la tolerancia a la frustración, dentro del espacio terapéutico se observan cambios aproximadamente a la quinta o sexta sesión trabajando bajo un esquema estructurado con una secuencia clara de actividades. Sin embargo, demora un poco más de tiempo en extrapolarse a su conducta en otros espacios donde no cuente con el soporte del terapeuta. En caso del colegio, muchas veces cuando existe una estructura de normas clara, el niño logra 
regularse a partir de lo aprendido en terapia y se adapta, lo cual se refleja positivamente en su desempeño. En el hogar, generalmente disminuyen las rabietas y conductas impulsivas pero suele necesitar cambios en la conducta de los padres o adultos responsables para lograr una modificación permanente de sus creencias hacia la intolerancia.

Del mismo modo, en los casos donde se presenta ansiedad, esta suele disminuir su frecuencia y los niños logran desenvolverse en espacios académicos y sociales de manera funcional, expresando sus pensamientos y actuando de forma espontánea acorde a sus deseos.

A lo largo de las intervenciones realizadas se encontró un factor en común en los niños que presentaban dificultades para tolerar la frustración acompañada de síntomas de ansiedad; este era la accesibilidad que tenían a los recursos electrónicos como celulares, tablets, TV o videojuegos. Lo cual, como parte de las pautas brindadas a los padres se les sugería disminuir el tiempo que los niños ocupaban ante estos estímulos. Cuando los padres cumplían dicha pauta y luego de algunas sesiones de intervención con los niños, se podían identificar cambios relevantes en su conducta.

A partir del análisis de lo trabajado se identifica una alta relación entre el éxito terapéutico con niños y el compromiso de sus padres. Tanto para que los niños cumplan con asistir a las sesiones de forma regular como para que se sigan las pautas y tareas que se encomiendan, las cuales influyen en gran porcentaje en el cumplimiento de los objetivos. Durante el 2018 el autor de este reporte brindó aproximadamente 30 sesiones de consejería psicológica emocional y conductual a padres de familia, colaborando en la mejora de la convivencia familiar en general y en el logro de objetivos terapéuticos de los niños.

De acuerdo a la experiencia realizada, se infiere que la psicoeducación pueda ser util para prevenir el desarrollo de problemas relacionados a la salud mental. Ya que, el recibir una psicoeducación en emociones (tanto en casa como en el colegio) que ahonde en la aceptación de uno mismo de forma incondicional, les daría herramientas para verbalizar lo que sienten y 
actuar sin sentirse juzgados. Lo cual, podría prevenir el desarrollo de conductas problema así como también evitar, en muchos casos, el desarrollo de patologías de salud mental más severas.

Según la información brindada por los padres que asisten a la institución, se puede afirmar que muchos padres de familia en la actualidad, por el ritmo de vida y la competitividad laboral, pasan la mayor parte de su tiempo fuera de casa. Por ello, el corto tiempo que pasan con sus hijos desean que sea agradable, limitándose a complacerlos en lo que pidan y sin espacios para enseñarles sobre la tolerancia o la importancia de las emociones negativas como fuente de cambio. Es muy común que estos padres recién reconozcan esta conducta complaciente inadecuada cuando su hijo presenta dificultades notorias. Es por ello que se consideraría de gran utilidad que existan más espacios para orientar a los padres en las diferentes instituciones educativas y de salud.

Finalmente, es importante mencionar que la metodología de trabajo de la institución aporta significativamente en el éxito de la intervención terapéutica en búsqueda del bienestar de quienes demandan ayuda. Pues, al tener sesiones programadas con los padres y formatos para intercambiar información con ellos y el colegio, permite recopilar los datos necesarios del entorno del niño y tener una visión integral del problema. Para luego poder organizar el plan de intervención con objetivos específicos según cada caso y actividades planteadas para realizar en un tiempo determinado, el cual será supervisado por coordinación antes y durante su aplicación. Ante la situación actual de salud mental en el Perú, descrita en el primer capítulo del presente reporte, sumada a la escasez de espacios y profesionales capacitados con estrategias convenientes para intervenir; se considera que será de gran utilidad el tomar en cuenta esta metodología para ser replicada por profesionales de la salud mental en otros espacios a lo largo de Lima y en un futuro en las diferentes regiones del país. 


\section{CONCLUSIONES}

A partir de la experiencia de trabajo realizada se ha podido llegar a las siguientes conclusiones:

- La metodología de la institución es de gran utilidad para trabajar la problemática que presentan los menores de edad que llegan a consulta, pues permite recopilar la información necesaria del ambiente familiar y académico, para luego organizar la intervención con objetivos específicos orientados a resultados visibles.

- Se ha podido comprobar que al trabajar en el proceso terapéutico de forma alineada con la familia y el colegio, los niños avanzan de forma más rápida hacia los objetivos terapéuticos, logrando cambios permanentes en su manera de interpretar las situaciones y desenvolverse en ellas.

- Las creencias irracionales mas comunes en los niños atendidos son la intolerancia a la frustración y la valoración condicional de uno mismo, las cuales suelen derivarse en diversas problemáticas emocionales que se reflejan en conductas disfuncionales en el ámbito social y académico.

- Se observa en repetidos casos, la falta de conocimiento de los padres en cuanto a métodos o estrategias de crianza que promuevan la estabilidad emocional en sus hijos.

- Se identificó que existe una relación entre el tiempo que pasan los niños frente a los estímulos tecnológicos visuales con la incidencia de ansiedad y dificultades para tolerar la frustración.

- Se ha podido comprobar la utilidad del modelo de terapia racional emotivo conductual (TREC) planteada por Albert Ellis, para intervenir en la problemática de los niños que fueron atendidos. 


\section{RECOMENDACIONES}

A continuación se detallaran las recomendaciones:

- Ante la problemática detallada se percibe la necesidad de propiciar mayores espacios de intervención terapéutica, así como de formar profesionales especializados en intervenir problemas de salud mental en todos los niveles socioeconómicos del país. Asimismo, sería de gran utilidad replicar la estructura de los programas terapéuticos individuales con niños del centro en otras instituciones del país.

- Se sugiere que al realizar una intervención terapéutica con niños se incluya una participación activa de los padres, manteniendo un compromiso con la asistencia del niño y las pautas que se encomiendan para aplicar en el hogar.

- Con el fin de prevenir la aparición de creencias irracionales en niños se propone que las instituciones educativas diseñen un currículo que incluya, desde nivel inicial, la Psicoeducación principalmente en reconocimiento y expresión de emociones, asi como la promoción de la aceptación incondicional de los seres humanos.

- Se recomienda que los padres manejen una estructura estable en casa, donde existan normas claras y horarios que permitan que los niños sigan una rutina. En la cual se asigne tiempo para comer, descansar, asearse, realizar deberes, jugar, entre otros.

- Se propone que tanto en el centro mencionado como en otras instituciones se realicen escuelas para padres o charlas de temas relevantes a fin de prevenir problemas comunes en cuanto a la crianza de sus hijos.

- En el trabajo descrito se ha podido observar una relación entre el tiempo frente a estímulos técnologicos y la recurrencia de síntomas de ansiedad e intolerancia a la frustración. Por lo tanto será relevante que se realicen investigaciones futuras en relación a este tema. 


\section{REFERENCIAS}

Alarcón, R. (2015). Salud mental y salud pública en el Perú: ya es tiempo de actuar. Revista de Neuro-Psiquiatría, 78(1), 1-2. $\quad$ Recuperado de http://www.scielo.org.pe/pdf/rnp/v78n1/a01v78n1.pdf

Browne, C., Dowd, T., \& Freeman, A. (2010). Rational and irrational beliefs; research, theory and clinical practice. Oxford University Press.

DiGiuseppe, R., Doyle, K., Dryden, W. \& Backx, W. (2014). A practitioner's guide to Rational Emotive Behavior Therapy (3rd ed.). Oxford University Press.

Dryden, W. (2009). Rational Emotive Behaviour Therapy. Londres: Routledge.

Ellis, A. y Tafrate, R. (2013). Controle su ira antes de que ella le controle a usted. Madrid: Editorial Paidós.

Organización Mundial de la Salud. (2013). Plan de acción integral sobre salud mental 20132020. Recuperado del sitio de internet de la Organización Mundial de la Salud https://www.who.int/mental_health/action_plan_2013/es/

Piazza, M. \& Fiestas, F. (2015). Cross sectional study of mental health services used in five cities of Peru. Salud Mental, 38(5), 337-345. doi: 10.17711/SM.0185-3325.2015.046 
